\title{
Fructose Malabsorption in Patients With Irritable Bowel Syndrome-like Symptoms: What Is the Role in the Pathogenesis and Clinical Implication?
}

\author{
Hye-Young Sung and Yong Sung Kim* \\ Division of Gastroenterology, Department of Internal Medicine, Digestive Disease Research Institute, Wonkwang University Sanbon Hospital, \\ Gunpo-si, Gyeonggi-do, Korea
}

\begin{abstract}
Article: Unclear abdominal discomfort: pivotal role of carbohydrate malabsorption
Goebel-Stengel M, Stengel A, Schmidtmann M, van der Voort I, Kobelt P, Mönnikes H.

(J Neurogastroenterol Motil 2014;20:228-235)
\end{abstract}

\section{Abdominal discomfort with negative medical tests is a com-} mon presentation in the gastrointestinal (GI) clinic, and it is usually diagnosed as irritable bowel syndrome (IBS). However, carbohydrate malabsorption, especially fructose malabsorption (FM), has been reported as another cause of unexplained abdominal symptoms, and also suggested to have a role in the pathogenesis of IBS. It has been reported that dietary fructose restriction and a low fermentable oligosaccharides, disaccharides, monosaccharides and polyols (FODMAPs) diet effectively reduced GI symptoms in patients with IBS, suggesting that these restrictions should be considered as a front-line therapy. ${ }^{1,2}$

In this issue, Goebel-Stengel et $\mathrm{al}^{3}$ reported a high prevalence of carbohydrate malabsorption in patients with IBS-like symptoms. Approximately $64 \%$ and $35 \%$ of the patients with IBS-like symptoms were symptomatic fructose and lactose malabsorbers, respectively. Although this was an observational study in a single center and not an interventional study, the number of enrolled patients was 2,390, making this the largest patient population study to date.

However, this result could not be applied directly to IBS because the patients in this study were not exactly diagnosed and classified by Rome criteria, but simply described as "patients with IBS-like symptoms." Even so, the results from this study suggest that the prevalence of FM is high in patients with unexplained abdominal symptoms, making this an important etiology in functional GI disorders (FGID) like IBS. It is worthy to note that the ability of the human small intestine to absorb fructose is physiologically limited, thus healthy populations also exhibit a high prevalence of FM ranging from $38 \%$ to $81 \%$, based on a dose of $50 \mathrm{~g}$ of fructose. ${ }^{4}$ Therefore, it seems that the prevalence of FM

Received: March 8, 2014 Revised: March 17, 2014 Accepted: March 18, 2014

(c) This is an Open Access article distributed under the terms of the Creative Commons Attribution Non-Commercial License (http://creativecommons. org/licenses/by-nc/3.0) which permits unrestricted non-commercial use, distribution, and reproduction in any medium, provided the original work is properly cited.

*Correspondence: Yong Sung Kim, MD, PhD

Department of Gastroenterology, Wonkwang University Sanbon Hospital, 321 Sanbon-ro, Gunpo-si, Gyeonggi-do 435-804, Korea

Tel: +82-31-390-2975, Fax: +82-31-398-2223, E-mail: wms89@hanmail.net

Financial support: This work was supported by Wonkwang University, 2012.

Conflicts of interest: None.

Author contributions: Hye-Young Sung: drafting the article; Yong Sung Kim: drafting and critical revising the article and final approval.

ORCID: Yong Sung Kim, http://orcid.org/0000-0001-8836-4818. 
is not different between healthy volunteers and patients with IBS or unexplained abdominal symptom, suggesting that FM is not a direct pathogenesis in the development of IBS. If so, what is the role of FM in the patients with IBS or "IBS-like symptoms"?

Unabsorbed fructose does not generate symptoms directly. Increased fluid retention produced by osmotic load, and gases produced by colonic bacteria fermentation distend the intestinal lumen, resulting in abdominal symptoms. ${ }^{4}$ Considering similar prevalence of healthy control and indirect mechanism of symptom generation, FM seems to act just as a triggering factor in subjects with underlying pathophysiology such as visceral hypersensitivity, small intestinal bacterial overgrowth, or abnormal colonic microbiota. Therefore, symptom development during a breath test is more frequently observed in patients with IBS than in control subjects. ${ }^{5}$ Even IBS patients without FM experience abdominal symptoms during a breath test. ${ }^{4}$ In this study by GoebelStengel et al, ${ }^{3} 10-30 \%$ of patients who reported abdominal symptoms during fructose and lactose breath tests had no increase in hydrogen levels. Conversely, healthy volunteers also experienced symptoms during high dose fructose breath tests. ${ }^{6}$

Because the hydrogen breath test has some accuracy issues and interpersonal variability problems, the data from it should be interpreted with caution. There is no consensus regarding the cut-off value for establishing a conclusive diagnosis of $\mathrm{FM},{ }^{4}$ and current guidelines do not recommend the routine use of this test in clinical practice. ${ }^{7}$ Positive symptoms without hydrogen increase in this study might be caused by the limitation of the breath test itself. Because unabsorbed carbohydrate would be fermented by bacteria in the colon, the amount of exhaled hydrogen depends on an individual's microbiota. Approximately 15-28\% of people do not produce hydrogen (so-called "hydrogen non-producers"), and so, the hydrogen exhaled during the test does not reach the detection-limit rate. ${ }^{4,8}$ Furthermore, slow intestinal transit time could produce a false negative result because hydrogen levels do not reach the detection-limit rate until end of the test. ${ }^{8}$ Test dose and concentration also differ in many studies. Goebel-Stengel et $\mathrm{al}^{3}$ used $50 \mathrm{~g}$ of fructose, but a dose of $25 \mathrm{~g}$ at a concentration of $10 \%$ more closely represents daily intake ${ }^{4}$, and healthy subjects can only absorb up to $25 \mathrm{~g}$ of fructose. ${ }^{6}$ The dose used in a fructose breath test can be selected according to the purpose of the test. ${ }^{4}$

How about the symptom correlation in FM? Certain types of IBS or symptoms might be related to specific carbohydrate malabsorption. Unfortunately, in this study, there were no data relating to subgroups of patients based on Rome criteria or a relationship between GI symptoms and the type of malabsorption. In another study which enrolled 1,372 FGID patients, fructose intolerance was more common than lactose intolerance in all FGID subgroups, but constipation-predominant IBS patients exhibited more lactose intolerance and less fructose intolerance than the others. ${ }^{9}$ So far, little data have been reported on whether specific GI symptoms are caused by FM. Considering the indirect mechanism of unabsorbed carbohydrates in symptom generation, theoretically, GI symptoms induced by FM may be nonspecific. In a study of 183 patients with unexplained abdominal symptoms, 134 patients had a positive fructose breath test. However, the prevalence of each symptom was not statistically different between positive-test and negative-test patients. ${ }^{10}$ In the study by GoebelStengel et al, ${ }^{3}$ severe fructose malabsorbers exhaled significantly higher hydrogen, but it is uncertain whether severe fructose malabsorbers can be classified as suffering from severe IBS-like symptoms because there is no data available to correlate with symptom severity. From previous reports, symptoms do not appear to correlate with the degree of hydrogen production. ${ }^{4}$

The study by Goebel-Stengel et $\mathrm{al}^{3}$ reaffirms the high prevalence of FM in a large population with unclear abdominal discomfort. Although it is not a direct etiology in IBS, this finding may justify performing hydrogen breath test to discriminate the carbohydrate malabsorption before diagnosing IBS rashly in patients with unexplained abdominal symptoms. However, the breath test has technical issues and is not available in every clinic. Moreover, it is still difficult to separate symptomatic carbohydrate malabsorber from IBS even after hydrogen breath test. Therefore, another predictor for discriminating carbohydrate malabsorption from IBS is needed. And also, future studies should be directed to investigate whether the breath test is mandatory before initiating dietary restriction in patient with IBS.

\section{References}

1. Shepherd SJ, Gibson PR. Fructose malabsorption and symptoms of irritable bowel syndrome: guidelines for effective dietary management. J Am Diet Assoc 2006;106:1631-1639.

2. Halmos EP, Power VA, Shepherd SJ, Gibson PR, Muir JG. A diet low in FODMAPs reduces symptoms of irritable bowel syndrome. Gastroenterology 2014;146:67-75, e5.

3. Goebel-Stengel M, Stengel A, Schmidtmann M, van der Voort I, Kobelt P, Mönnikes H. Unclear abdominal discomfort - pivotal role of carbohydrate malabsorption. J Neurogastroenterol Motil 2014;20: 228-235.

4. Gibson PR, Newnham E, Barrett JS, Shepherd SJ, Muir JG. Review article: fructose malabsorption and the bigger picture. Aliment Pharmacol Ther 2007;25:349-363.

5. Nelis GF, Vermeeren MA, Jansen W. Role of fructose-sorbitol mal- 
absorption in the irritable bowel syndrome. Gastroenterology 1990; 99:1016-1020.

6. Rao SS, Attaluri A, Anderson L, Stumbo P. Ability of the normal human small intestine to absorb fructose: evaluation by breath testing. Clin Gastroenterol Hepatol 2007;5:959-963.

7. Gasbarrini A, Corazza GR, Gasbarrini G, et al. Methodology and indications of $\mathrm{H} 2$-breath testing in gastrointestinal diseases: the Rome Consensus Conference. Aliment Pharmacol Ther 2009;29 (suppl 1):1-49.

8. D'Angelo G, Di Rienzo TA, Scaldaferri F, et al. Tricks for interpret- ing and making a good report on hydrogen and ${ }^{13} \mathrm{C}$ breath tests. Eur Rev Med Pharmacol Sci 2013;17(suppl 2):90-98.

9. Wilder-Smith $\mathrm{CH}$, Materna A, Wermelinger $\mathrm{C}$, Schuler J. Fructose and lactose intolerance and malabsorption testing: the relationship with symptoms in functional gastrointestinal disorders. Aliment Pharmacol Ther 2013;37:1074-1083.

10. Choi YK, Johlin FC Jr, Summers RW, Jackson M, Rao SS. Fructose intolerance: an under-recognized problem. Am J Gastroenterol 2003; 98:1348-1353. 